\title{
Exciton-phonon coupling in single quantum dots with different barriers
}

\author{
Daniel Dufåker, L. O. Mereni, Fredrik K. Karlsson, V. Dimastrodonato, \\ G. Juska, Per-Olof Holtz and E. Pelucchi
}

\section{Linköping University Post Print}

N.B.: When citing this work, cite the original article.

Original Publication:

Daniel Dufåker, L. O. Mereni, Fredrik K. Karlsson, V. Dimastrodonato, G. Juska, Per-Olof Holtz and E. Pelucchi, Exciton-phonon coupling in single quantum dots with different barriers, 2011, Applied Physics Letters, (98), 25, 251911.

http://dx.doi.org/10.1063/1.3600781

Copyright: American Institute of Physics http://www.aip.org/

Postprint available at: Linköping University Electronic Press

http://urn.kb.se/resolve?urn=urn:nbn:se:liu:diva-67198 


\title{
Exciton-phonon coupling in single quantum dots with different barriers
}

\author{
D. Dufåker, ${ }^{1, a)}$ L. O. Mereni, ${ }^{2}$ K. F. Karlsson, ${ }^{1}$ V. Dimastrodonato, ${ }^{2}$ G. Juska, ${ }^{2}$ \\ P. O. Holtz, ${ }^{1}$ and E. Pelucchi ${ }^{2}$ \\ ${ }^{1}$ Department of Physics, Chemistry and Biology, Linköping University, 58183 Linköping, Sweden \\ ${ }^{2}$ Tyndall National Institute, University College Cork, Cork, Ireland
}

(Received 2 March 2011; accepted 26 May 2011; published online 22 June 2011)

\begin{abstract}
The coupling between longitudinal-optical (LO) phonons and neutral excitons in two different kinds of InGaAs pyramidal quantum dots (QDs) embedded in either AlGaAs or GaAs barriers is experimentally examined. We find a slightly weaker exciton-LO-phonon coupling and increased linewidth of the phonon replicas for the QDs with GaAs barriers compared to the ones with AlGaAs barriers. These results, combined with the fact that the LO-phonon energy of the exciton is the same for both kinds of dots, are taken as evidence that the excitons mainly couple to LO-phonons within the QDs. (C) 2011 American Institute of Physics. [doi:10.1063/1.3600781]
\end{abstract}

A quantum dot (QD) is commonly referred to as an artificial atom due to the discreteness of its energy levels. ${ }^{1}$ Charge carriers trapped in the QD, following optical excitation, form different kinds of exciton complexes depending on the number of interacting electrons $(e)$ and holes $(h)$. There is a nonzero probability that some energy remains in the lattice, in the form of quantized lattice vibrations, after the optical recombination of these excitonic complexes. In the optical recombination spectra this is typically manifested by the presence of phonon replicas at LO-phonon energies, $\hbar \omega_{\mathrm{LO}}$, below the zero-phonon emission. The strength of the phonon replicas is described by the Huang-Rhys ${ }^{2}$ parameter, which can be experimentally determined as the intensity ratio between the first and zeroth order phonon emission. For the neutral exciton $(X=1 e+1 h)$, the Huang-Rhys parameter is directly related to the exciton-phonon coupling strength determined by Fröhlich interactions. ${ }^{3}$

Until recently there have been a very limited number of papers on longitudinal-optical (LO)-phonon coupling of single QDs, mainly restricted to the neutral exciton and biexciton $(2 X=2 e+2 h){ }^{4-6}$ The effect of additional charge was theoretically predicted to enhance the Huang-Rhys parameter in GaAs microcrystallites. ${ }^{3}$ Accordingly, a weak first order LO-phonon replica was interpreted as a sign of QD charge neutrality. ${ }^{4}$ Nevertheless, it was recently shown experimentally and theoretically that the Huang-Rhys parameter for single InGaAs/AlGaAs QDs was significantly reduced for the positively charged exciton $\left(X^{+}=1 e+2 h\right)$ compared to $X$ and the negatively charged exciton $\left(X^{-}=2 e+1 h\right){ }^{7}$ Furthermore, it was concluded that the exciton complexes couple to LO-phonons either in the QDs or in the vertical quantum wires (VQWRs) in contact with the QDs. ${ }^{7}$

In this letter, we present an experimental comparison of the exciton-LO-phonon coupling in two types of pyramidal QDs, $\mathrm{In}_{0.15} \mathrm{Ga}_{0.85} \mathrm{As} / \mathrm{AlGaAs}$ dots and $\mathrm{In}_{0.25} \mathrm{Ga}_{0.75} \mathrm{As} / \mathrm{GaAs}$ dots. These pyramidal QDs are of particular interest since their potentially high symmetry is important for the generation of entangled photons, as demonstrated in a similar site controlled system. ${ }^{8-10}$ LO-phonon mediated interaction of QD states with the surrounding matrix contributes to dephasing which degrade the quality of entanglement. ${ }^{11}$ In order to

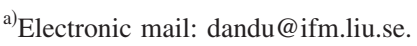

determine the origin of the LO-phonon coupling, within the QD or in the VQWR, two specially designed samples were fabricated, with and without a VQWR, respectively. The results of our comparison of the two QD systems point out that the coupling between excitons and LO-phonons mainly occur in the QD itself and not in the VQWR. The slightly lower value of the Huang-Rhys parameter for the exciton, $X$, and the increased linewidth of the phonon replicas for the $\mathrm{In}_{0.25} \mathrm{Ga}_{0.75} \mathrm{As} / \mathrm{GaAs}$ dots compared to the $\mathrm{In}_{0.15} \mathrm{Ga}_{0.85} \mathrm{As} /$ AlGaAs dots are also discussed.

In this experimental study, the two different samples used were grown by low pressure metal organic chemical vapor deposition on a patterned GaAs (111)B substrate, in a system, where particular care is taken in monitoring unintentional impurity levels. ${ }^{12,13}$ The pattern consists of inverted tetrahedral micropyramids with a $7.5 \mu \mathrm{m}$ pitch. The dots self-assemble at the tip of the inverted tetrahedral recesses during the deposition of the $\mathrm{In}_{\mathrm{x}} \mathrm{Ga}_{1-\mathrm{x}}$ As layer, sandwiched between two barrier layers, thanks to capillarity effects and decomposition rate anisotropy. ${ }^{14,15}$

The first type of QDs is formed from an $\mathrm{In}_{0.15} \mathrm{Ga}_{0.85} \mathrm{As}$ layer, nominally $0.8 \mathrm{~nm}$ thick, surrounded by $\mathrm{Al}_{0.3} \mathrm{Ga}_{0.7} \mathrm{As}$ barrier layers. For this type, a VQWR and three vertical quantum wells (VQWs) are formed at the center of the QD due to alloy segregation in the barriers. ${ }^{16}$ Modeling of this type of QD as a disk with height (diameter) $6 \mathrm{~nm}(24 \mathrm{~nm})$ yields the computed exciton energy in consistency with measurements. ${ }^{7}$ The second type of QDs is formed from an $\mathrm{In}_{0.25} \mathrm{Ga}_{0.75}$ As layer, nominally $0.5 \mathrm{~nm}$ thick, surrounded by GaAs barrier layers. In this sample, there is no formation of a VQWR or VQWs since no alloyed material is used in the barrier layers. The thinner InGaAs QD layer for the second type of QDs in combination with a wider self-limiting profile for GaAs barriers, ${ }^{19}$ as compared to $\mathrm{Al}_{0.3} \mathrm{Ga}_{0.7} \mathrm{As}$, result in an estimated dot height (diameter) of about $\sim 3 \mathrm{~nm}$ $(\sim 36 \mathrm{~nm})$. However, additional structural analysis will be needed to verify these estimates. The insets of Figs. 1(a) and 1(b) show models of the cleaved pyramid unveiling the different QD structures used in the experiments. The samples were back-etched after growth in order to increase the efficiency of light extraction. ${ }^{17-19}$

The QDs were excited individually by a Ti-sapphire laser (wavelength $732 \mathrm{~nm}$ ) in a microphotoluminescence 


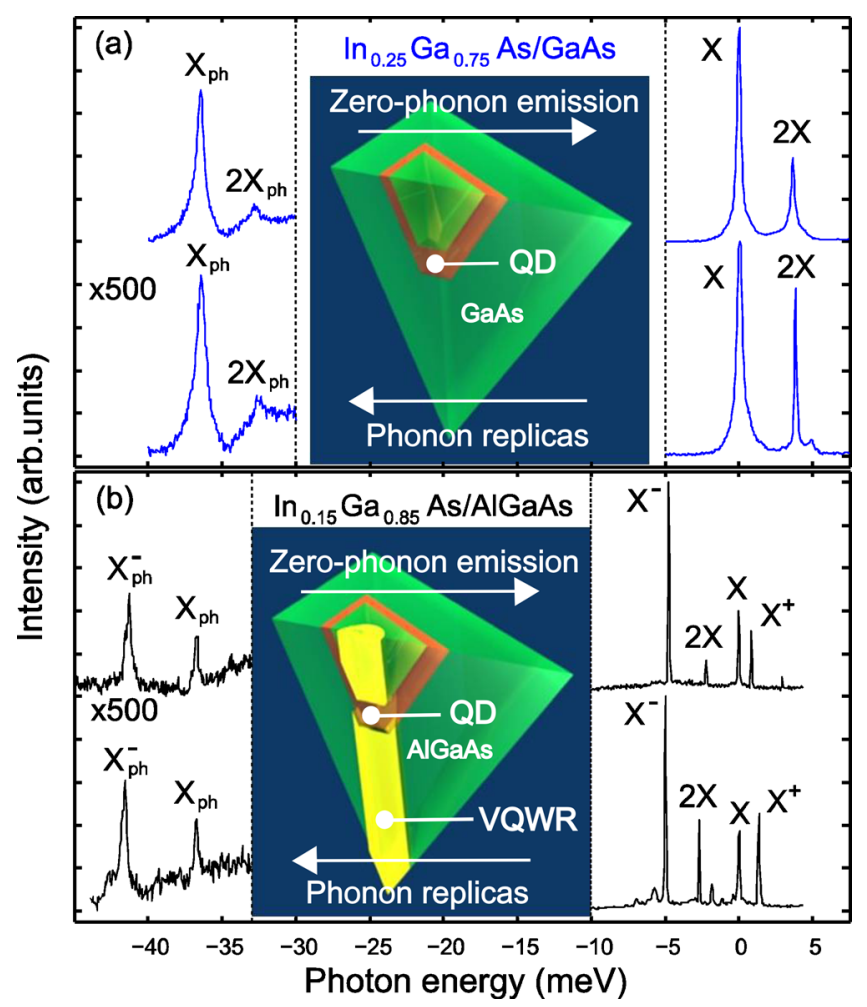

FIG. 1. (Color online) $\mu \mathrm{PL}$ spectra of the zero-phonon emission and the corresponding first order LO-phonon replicas (enhanced 500 times) for (a) two different $\operatorname{In}_{0.25} \mathrm{Ga}_{0.75} \mathrm{As} / \mathrm{GaAs}$ QDs and (b) two different $\mathrm{In}_{0.15} \mathrm{Ga}_{0.85} \mathrm{As} / \mathrm{AlGaAs}$ QDs. For all spectra, the energy of $X$ (about 1440 $\mathrm{meV}$ for both QD types) is set to zero. The insets show models of the cleaved pyramid unveiling the different QD structures.

( $\mu \mathrm{PL})$ setup, where the samples were kept at a temperature of $4 \mathrm{~K}$ (in one case $30 \mathrm{~K}$ ). A single grating monochromator (1200 grooves $/ \mathrm{mm}$ blazed for $750 \mathrm{~nm}$, focal length $0.55 \mathrm{~m}$ ) with a spectral resolution of $\sim 0.1 \mathrm{meV}$ equipped with a charge coupled device (CCD) camera was used to record the spectra from single QDs. The zeroth and first order phonon emissions, differing in intensity by about three orders in magnitude, were detected simultaneously by covering a part of the CCD-chip with a neutral density filter transmitting $1.46 \%$. The part shaded by the filter recorded the zerophonon emission.

The excitation conditions, such as excitation power and crystal temperature, determines the average number of electrons and holes populating the QDs. For the $\mathrm{Al}_{0.3} \mathrm{Ga}_{0.7} \mathrm{As}$ barrier sample, the different exciton species in the spectra were identified through comparison with the data available for similar QDs. ${ }^{20-22}$ For the $\mathrm{In}_{0.25} \mathrm{Ga}_{0.75} \mathrm{As} / \mathrm{GaAs}$ QDs, the biexciton exhibits negative binding energy and power dependence and time-resolved PL measurements were performed in order to verify the assignment of the exciton and the biexciton. The biexciton shows the expected quadratic power dependence in PL and faster recombination in time-resolved PL. ${ }^{23,24}$ The exciton decay is also, as expected, delayed compared to the biexciton. ${ }^{21}$

The average phonon energies for the different exciton species were determined for both types of dot samples (see Fig. 1). The average LO-phonon energies for the exciton were in both cases determined to be: $\hbar \omega(X)_{\mathrm{LO}}=36.3 \mathrm{meV}$ (average from 10 QDs). For completeness, we also include the average LO-phonon energies determined for other exci-

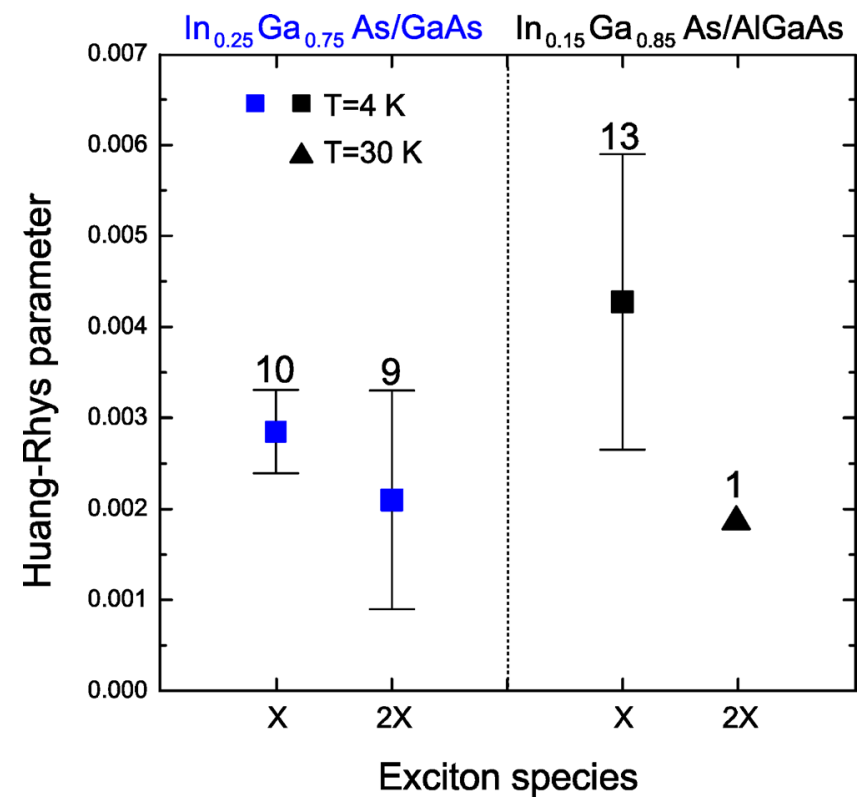

FIG. 2. (Color online) The measured Huang-Rhys parameter represented by the mean values of in total 10 (13) different $\operatorname{In}_{0.25} \mathrm{Ga}_{0.75} \mathrm{As} / \mathrm{GaAs}$ $\left(\mathrm{In}_{0.15} \mathrm{Ga}_{0.85} \mathrm{As} / \mathrm{AlGaAs}\right)$ QDs. The bars indicate the standard deviation from the mean, and the numbers above indicate the number of measured QDs. In order to facilitate measurements of the biexciton phonon replica for the $\mathrm{In}_{0.15} \mathrm{Ga}_{0.85} \mathrm{As} / \mathrm{AlGaAs}$ QDs, the temperature had to be raised to $30 \mathrm{~K}$.

ton species: $\hbar \omega(2 X)_{\mathrm{LO}}=36.4 \mathrm{meV}$ for $\mathrm{In}_{0.25} \mathrm{Ga}_{0.75} \mathrm{As} / \mathrm{GaAs}$ QDs and $\hbar \omega\left(X^{-}\right)_{\mathrm{LO}}=36.0 \mathrm{meV}$ for $\mathrm{In}_{0.15} \mathrm{Ga}_{0.85} \mathrm{As} / \mathrm{AlGaAs}$ QDs.

The Huang-Rhys parameter for $X$ and $2 X$ was determined for in total 10 (13) different $\mathrm{In}_{0.25} \mathrm{Ga}_{0.75} / \mathrm{GaAs}$ $\left(\mathrm{In}_{0.15} \mathrm{Ga}_{0.85} \mathrm{As} / \mathrm{AlGaAs}\right)$ QDs. Spectra like those in Fig. 1 were used to determine the integrated peak intensity (summation of the data points for each peak after background removal) and the Huang-Rhys parameter was determined as the ratio between the integrated intensities of the phonon replica and the corresponding zero-phonon emission. The extracted average values for each type of QD are displayed in Fig. 2. As seen in Fig. 2, the Huang-Rhys parameter for the exciton, $X$, is slightly lower for the $\operatorname{In}_{0.25} \mathrm{Ga}_{0.75} \mathrm{As} / \mathrm{GaAs}$ QDs compared to the $\mathrm{In}_{0.15} \mathrm{Ga}_{0.85} \mathrm{As} / \mathrm{AlGaAs}$ QDs. This may seem puzzling at first glance, since raising the Inconcentration in the QD increases the strain as well as the strain induced piezoelectric field, which further separates the hole and electron in the QD and thereby enhances the exciton LO-phonon coupling. ${ }^{3}$ However, the GaAs barrier dot is thinner than its $\mathrm{Al}_{0.3} \mathrm{Ga}_{0.7} \mathrm{As}$ counterpart (nominally 0.5 and $0.8 \mathrm{~nm}$, respectively, and the shape of the self limited profile of the two different kinds of barrier further magnifies this difference ${ }^{16}$ ), limiting the carrier separation caused by the piezoelectric field. For the biexciton, $2 X$, the strengths of the replicas are similar for both types of QDs.

For both types of QDs, the full width at half maximum (FWHM) of the zero-phonon emission and the first order LO-phonon replicas are plotted in Fig. 3, as determined by Voigt peak fitting showing a considerably larger linewidth for the replicas compared to the zero-phonon emission. In particular for the exciton, $X$, there is a broadening of $\sim 380 \mu \mathrm{eV}$ for the $\operatorname{In}_{0.25} \mathrm{Ga}_{0.75} \mathrm{As} / \mathrm{GaAs}$ QDs and $\sim 230 \mu \mathrm{eV}$ for the $\operatorname{In}_{0.15} \mathrm{Ga}_{0.85} \mathrm{As} / \mathrm{AlGaAs}$ QDs. Thus, there is an additional broadening of the exciton replicas for the 


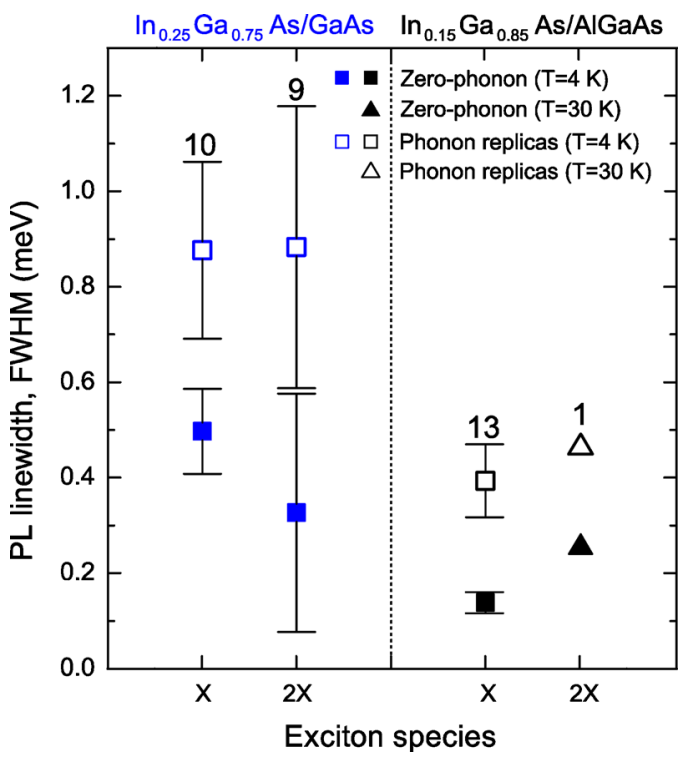

FIG. 3. (Color online) Measured linewidths (FWHM) represented by the mean values of in total 10 (13) different $\mathrm{In}_{0.25} \mathrm{Ga}_{0.75} \mathrm{As} / \mathrm{GaAs}$ $\left(\mathrm{In}_{0.15} \mathrm{Ga}_{0.85} \mathrm{As} / \mathrm{AlGaAs}\right)$ QDs. The bars indicate the standard deviation from the mean, and the numbers above indicate the number of measured QDs. In order to facilitate measurements of the biexciton phonon replica for the $\mathrm{In}_{0.15} \mathrm{Ga}_{0.85} \mathrm{As} / \mathrm{AlGaAs}$ QDs, the temperature had to be raised to $30 \mathrm{~K}$.

$\mathrm{In}_{0.25} \mathrm{Ga}_{0.75} \mathrm{As} / \mathrm{GaAs}$ QDs of $\sim 150 \mu \mathrm{eV}$ compared to the $\mathrm{In}_{0.15} \mathrm{Ga}_{0.85} \mathrm{As} / \mathrm{AlGaAs}$ QDs.

The exciton LO-phonon coupling has, as previously mentioned, been determined to occur either (1) in the VQWRs and/or (2) within the QDs. ${ }^{7}$ In case (1), the replacement of the AlGaAs barrier by GaAs should shift the low temperature LO-phonon energy from $36.3 \mathrm{meV}$ (corresponding to the $\mathrm{Al}_{0.04} \mathrm{Ga}_{0.96} \mathrm{As}$ of the VQWR) to the bulk GaAs value of $36.6 \mathrm{meV}{ }^{25}$ In addition, there should also be a reduced FWHM of the replicas, since broadening attributed to alloy disorder and composition variations vanishes for GaAs barriers, leaving merely minor broadening mechanisms related to the intrinsic GaAs LO-phonon lifetime $(\sim 70 \mu \mathrm{eV})$ and the GaAs bulk phonon dispersion (less than $50 \mu \mathrm{eV}){ }^{26,7}$ This is, however, contradicted by the experimental observations, where instead the LO-phonon energy remains at $36.3 \mathrm{meV}$ also for the QDs with GaAs barriers, and there is an additional broadening of the phonon replicas of $\sim 150 \mu \mathrm{eV}$. In case (2), on the other hand, the excitons couple to LO-phonons within the QDs. The LO-phonon energy is then strongly dependent on the In-composition of the QD but unaffected by the barrier composition, increasing the QD In-content from 15\% (the first QD type) to $25 \%$ the (second QD type) which downshifts the LO-phonon energy by $\sim 0.5 \mathrm{meV}$, but the correspondingly increased compressive strain upshifts the phonon energy by $\sim 0.4-0.7 \mathrm{meV}$, as determined from the theory with parameters employed from Refs. 27 and 28, and the QD model from Ref. 7. Due to these competing mechanisms, the mean LO-phonon energy is expected to remain essentially the same for both QD types in case (2), while the enhanced strain induced splitting of the LO-phonon modes and variations in alloy composition for higher In-composition explains the additional broadening observed for the replicas in the most In-rich QD type.

In conclusion, it is found that different kinds of InGaAs QDs with either GaAs or AlGaAs barriers exhibit identical LO-phonon energies $(36.3 \mathrm{meV})$ and very similar exciton-
LO-phonon Fröhlich coupling strength (Huang-Rhys parameter for $X$ : 0.003-0.004). The exciton couples to LO-phonons mainly within the QD, and the slightly lower value of the Huang-Rhys parameter observed for the $\mathrm{In}_{0.25} \mathrm{Ga}_{0.75} \mathrm{As}$ dots is attributed to the fact that these In-rich QDs are thinner, as compared to the other type of investigated $\mathrm{In}_{0.15} \mathrm{Ga}_{0.85} \mathrm{As}$ dots, limiting the possibility of charge separation within the QD.

This research was enabled by the Irish Higher Education Authority Program for Research in Third Level Institutions (2007-2011) via the INSPIRE program, by Science Foundation Ireland under Grant Nos. 05/IN.1/I25 and 08/RFP/MTR/ 1659, and by grants from the Swedish Research Council (VR) and by equipment grants from the K. A. Wallenberg Foundation. We are grateful to Dr. K. Thomas for his support with the MOVPE system. D.D. gratefully acknowledges financial support from the Font-D, at Linköping University.

${ }^{1}$ D. Gammon, Nature (London) 405, 899 (2000).

${ }^{2}$ K. Huang, and A. Rhys, Proc. R. Soc. London, Ser. A 204, 406 (1950).

${ }^{3}$ S. Nomura and T. Kobayashi, Phys. Rev. B 45, 1305 (1992).

${ }^{4}$ R. Heitz, I. Mukhametzhanov, O. Stier, A. Madhukar, and D. Bimberg, Phys. Rev. Lett. 83, 4654 (1999).

${ }^{5}$ P. Hawrylak, G. A. Narvaez, M. Bayer, and A. Forchel, Phys. Rev. Lett. 85, 389 (2000)

${ }^{6}$ F. Gindele, K. Hild, W. Langbein, and U. Woggon, Phys. Rev. B 60 , R2157 (1999).

${ }^{7}$ D. Dufåker, K. F. Karlsson, V. Dimastrodonato, L. O. Mereni, B. E. Sernelius, P. O. Holtz, and E. Pelucchi, Phys. Rev. B 82, 205421 (2010).

${ }^{8}$ K. F. Karlsson, M. A. Dupertuis, D. Y. Oberli, E. Pelucchi, A. Rudra, P. O. Holtz, and E. Kapon, Phys. Rev. B 81, 161307(R) (2010).

${ }^{9}$ K. F. Karlsson, V. Troncale, D. Y. Oberli, A. Malko, E. Pelucchi, A. Rudra, and E. Kapon, Appl. Phys. Lett. 89, 251113 (2006).

${ }^{10}$ A. Mohan, M. Felici, P. Gallo, B. Dwir, A. Rudra, J. Faist, and E. Kapon, Nat. Photonics 4, 302 (2010).

${ }^{11}$ A. Carmele, F. Milde, M.-R. Dachner, M. Bagheri Harouni, R. Roknizadeh, M. Richter, and A. Knorr, Phys. Rev. B 81, 195319 (2010).

${ }^{12}$ E. Pelucchi, N. Moret, B. Dwir, D. Y. Oberli, A. Rudra, N. Gogneau, A. Kumar, E. Kapon, E. Levy, and A. Palevski, J. Appl. Phys. 99, 093515 (2006).

${ }^{13}$ V. Dimastrodonato, L. Mereni, R. J. Young, and E. Pelucchi, J. Cryst. Growth 312, 3057 (2010).

${ }^{14}$ E. Pelucchi, V. Dimastrodonato, A. Rudra, K. Leifer, E. Kapon, L. Bethke, P. Zestanakis, and D. Vvedenski, Phys. Rev. B 83, 205409 (2011).

${ }^{15}$ E. Pelucchi, S. Watanabe, K. Leifer, Q. Zhu, B. Dwir, P. D. L. Rios, and E. Kapon, Nano Lett. 7, 1282 (2007).

${ }^{16}$ Q. Zhu, E. Pelucchi, S. Dalessi, K. Leifer, M.-A. Dupertuis, and E. Kapon, Nano Lett. 6, 1036 (2006).

${ }^{17}$ A. Hartmann, Y. Ducommun, K. Leifer, and E. Kapon, J. Phys.: Condens Matter 11, 5901 (1999).

${ }^{18}$ L. O. Mereni, V. Dimastrodonato, R. J. Young, and E. Pelucchi, Appl. Phys. Lett. 94, 223121 (2009).

${ }^{19}$ V. Dimastrodonato, L. O. Mereni, R. J. Young, and E. Pelucchi, Phys. Status Solidi B 247, 1862 (2010).

${ }^{20}$ A. Hartmann, Y. Ducommun, E. Kapon, U. Hohenester, and E. Molinari, Phys. Rev. Lett. 84, 5648 (2000).

${ }^{21}$ V. Troncale, K. F. Karlsson, D. Y. Oberli, M. Byszewski, A. Malko, E. Pelucchi, A. Rudra, and E. Kapon, J. Appl. Phys. 101, 081703 (2007).

${ }^{22}$ M. H. Baier, A. Malko, E. Pelucchi, D. Y. Oberli, and E. Kapon, Phys. Rev. B 73, 205321 (2006)

${ }^{23}$ K. Brunner, G. Abstreiter, G. Böhm, G. Tränkle, and G. Weimann, Phys. Rev. Lett. 73, 1138 (1994).

${ }^{24}$ R. M. Thompson, R. M. Stevenson, A. J. Shields, I. Farrer, C. J. Lobo, D. A. Ritchie, and M. L. Leadbetter, Phys. Rev. B 64, 201302(R) (2001).

${ }^{25}$ G. Irmer, M. Wenzel, and J. Monecke, Phys. Status Solidi B 195, 85 (1996).

${ }^{26}$ B. Jusserand and J. Sapriel, Phys. Rev. B 24, 7194 (1981).

${ }^{27}$ F. Cerdeira, C. J. Buchenauer, F. H. Pollak, and M. Cardona, Phys. Rev. B 5, 580 (1972).

${ }^{28}$ P. Wickboldt, E. Anastassakis, R. Sauer, and M. Cardona, Phys. Rev. B 35, 1362 (1987). 